\title{
L'écriture du cri chez Henry Bauchau
}

Les plus valables de mes pensées maintenant sont presque des cris. ${ }^{432}$

Comment l'écriture du cri s'ancre-t-elle dans les premiers textes de Henry Bauchau, ceux qui correspondent à sa période suisse, pour se développer dans les scènes de cri marquantes narrées dans Edipe sur la route, Antigone ou Le Boulevard périphérique? Cet article propose quelques réponses à cette interrogation, en commençant par suggérer certains repères dans les œuvres premières, pour se concentrer ensuite plus particulièrement sur les textes de la maturité littéraire de Bauchau. Il sera question de la construction du personnage à travers le cri, et du paradoxe du cri mis en langue, du cri écrit, dans la mesure où ce qui tient de l'inarticulé (le cri) doit être articulé (par la langue), et s'offre comme potentiel polysémique. À travers ce parcours d'un motif, nous approcherons des notions pertinentes dans la poétique bauchalienne, telles que la sidération et l'espérance. Nous nous en tiendrons ici à une démarche thématique, bien qu'elle puisse ouvrir à une réflexion à propos du cri comme mode d'énonciation.

Précisons d'emblée que nous ne considérons pas l'écriture du cri chez Bauchau comme Alain Marc a pu le faire chez d'autres auteurs (Sade, Bataille, Noël, Guyotat... ${ }^{433}$. L'essai dans lequel ces réflexions prennent forme se propose de considérer l'écriture du cri comme une grille de relecture de l'ensemble de la littérature, presque comme une catégorie littéraire, qui serait celle d'une littérature de la transgression, et notamment d'une littérature pornographique. Chez Bauchau, ce n'est pas l'expression en tant que cri qui importe, mais le cri même, la mise en récit du cri et de ce qu'il exprime. Une des différences essentielles, dans cette perspective, est que ce dont parle Alain Marc est une virtualité du désespoir et une forme de destruction, tandis que le cri chez Bauchau est l'expression d'une espérance, d'un lien entre les êtres et leurs profondeurs.

Dans Géologie, le premier recueil de poésie de Bauchau, se fait entendre le cri de l'origine, celui du nouveau-né:

432 Henry Bauchau, Passage de la Bonne-Graine. Journal (1997-2001), Arles, Actes Sud, 2002, p. 13. 433 Alain Marc, L'Écriture du cri. (Sade, Bataille, Maïakovski, Mansour, Giauque, Venaille, Laâbi, Calaferte, Noël, Guyotat...), Orléans, L'Écarlate, 2000. 
Je vis d'être chassé mais ma révolte est grande.

J'étouffe donc je suis, je crie, je suis au monde.

Ô ma mère qui m'as effrayé, je te quitte. ${ }^{434}$

Il est accompagné de ceux de la nature, qui en devient anthropomorphe: "cris

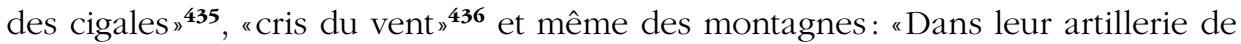
soleils, que crient-elles / Que crient-elles si haut que je ne puis l'entendre?»437. Lêtre vient au monde en criant, comme l'écrivain trouve son avènement dans la prime écriture pour interpréter le monde qui l'entoure.

Dans L'Escalier bleu, c'est le cri de l'enfance qui prend corps : «Et l'on rêvait des cris, tendres cris des surprises ${ }^{\mathbf{4 3 8}}$; celui des amants aussi, dans "Liant déliant» et plus particulièrement dans le poème "Déchire ô Verdoyant»:
Frappant
frappant contre ton nom
ne sachant plus qu'attendre
et crier tes syllabes à voix basse
J'oublie ton visage
je perds le son de ta voix ${ }^{439}$

Il y a aussi les amants du poème «Vivant ne vivant plus»:

Criant ne criant plus

la bouche enfoncée dans la nuit

ils roulent sur l'oreiller impossible du temps

et c'est le temps qui les nourrit ${ }^{440}$

Après le cri de l'origine, c'est donc celui de l'enfance, puis celui de l'amour, qui prend forme, dans la tension de l'oxymore (crier "à voix basse») et du participe présent sitôt nié (criant "ne criant plus»). Ces formes de l'écriture participent de l'expérience de la constitution de soi du sujet, pour lequel le cri et le sens ne font qu'un.

Dans les premiers textes de théâtre de Bauchau, Gengis Khan et La Machination (pièce intitulée plus tard La Reine en amont), le cri prend d'autres formes. Dans Gengis Khan, il s'agit de relier les personnages aux cieux, au désir de puissance, au souffle épique qui traverse la pièce. Dans le premier tableau, Temoudjin (qui deviendra Gengis Khan) dit à Timour: "Tu es le premier à me parler ainsi. Mon nom n'avait pas encore été appelé et tu viens de le crier aux étoiles!»41. Dans le

434 Henry Bauchau, Géologie, Paris, Gallimard, 1958, p. 114.

435 Ibid., p. 73.

436 Ibid., p. 110

437 Ibid., p. 113.

438 Henry Bauchau, L'Escalier bleu, Paris, Gallimard, 1964, p. 12.

439 Ibid., p. 57.

440 Ibid., p. 58.

441 Henry Bauchau, Gengis Khan, dans Théâtre complet, Arles, Actes Sud, coll. Papiers, 2001, Premier tableau, Scène 1, p. 72. 
deuxième tableau, il affirme: "J'ai rassemblé les Mongols et je leur ai posé cette question: Voulez-vous être un seul peuple et m'avoir pour chef, ou voulez-vous être plusieurs peuples? Ils ont levé leurs armes, ils ont crié: "Nous serons un seul peuple et toi, Temoudjin, notre chef!” "\$42. Chaque tableau se trouve ainsi marqué par un cri, celui de la conquête, celui de l'intensité des sentiments, celui de la révolte des asservis.

Dans La Machination, le cri constitue un des savoirs convoités par Alexandre pour égaler ou plutôt surpasser son père Philippe:

(Alexandre profite [d'un] instant d'inattention de son père pour lui saisir la main et tenter de la tordre. Philippe se laisse faire, puis relève la main. Alexandre la mord. Philippe pousse un grand cri. Alexandre, surpris, tombe à la renverse. Philippe le relève et le cajole.)

Philippe: Alors, on tombe pour un cri?

Alexandre: Tu m'apprendras ce cri? ${ }^{443}$

Il est aussi conçu comme arme de conquête amoureuse entre Laïos et Jocaste, au troisième acte:

Jocaste: Dans le taillis, j'ai entendu un bruit de bête. Moi, qui n'avais jamais eu peur, l'effroi m'a prise aux genoux. Je me suis enfuie. J'ai entendu un cri et je me suis évanouie. [...] Tu es revenu le soir, tu m’apportais mes premières sandales dorées et un cheval. En le voyant, j’ai su que tu m'aimais.

Laïos: Moi je l'ai su dès que je t'ai entendue. J'avais fait route toute la nuit et je m'étais endormi, armé. Quand tu t'es enfuie, si légère, j’ai pensé que je ne pourrais pas te rattraper.

Jocaste: Et tu as poussé ce terrible cri. ${ }^{444}$

Ainsi le cri est-il considéré comme un instrument de sidération, et par conséquent de pouvoir.

Dans les deux premiers romans de Bauchau, La Déchirure et Le Régiment noir, le motif du cri est présent, mais n'est pas développé en des scènes aussi marquantes que dans les romans du cycle thébain ou dans Le Boulevard périphérique. Dans La Déchirure, qui est le roman de l'analyse, de la mort de la mère, de l'enfance et de ses scènes primitives, les cris sont ceux du narrateur enfant ou de la mère mourante. L'enfant hurle, terrorisé par l'incendie de Louvain qui le sépare de sa mère ${ }^{445}$, ou désemparé par sa nouvelle maison ${ }^{446}$; il exulte à toute nouvelle sensation, notamment érotique ${ }^{447}$. En contrepoint, le cri de la mère en agonie, se débattant pour survivre avec le peu de souffle qui lui reste, se transforme en râle

442 Ibid., Deuxième tableau, Scène 2, p. 77.

443 Henry Bauchau, La Machination, Lausanne, L’Aire, 1969, Prologue, p. 19.

444 Ibid., Acte III, Scène 2, p. 111.

445 Henry Bauchau, La Déchirure, Bruxelles, Labor, 1998, p. 44 et suivantes.

446 Ibid., pp. 127-128.

447 Ibid., p. 200. 
avant de s'éteindre ${ }^{\mathbf{4 4 8}}$. L'écriture elle-même, ce "levier de l'analyse „449, rejoue ces émotions :

J'ai tellement résisté. J'ai tellement refusé ce qui m’arrive que cette fois je suis ouvert. Je suis fendu en deux par le besoin de crier: Ha! Rien que: Ha! comme un bûcheron. Et cette syllabe contient la naissance, les ténèbres et la signification suffisante de la vie. C'est en vain que je voudrais capter le cri d'existence des voyelles. Ce qui était dictée abrupte, langage absolu, ne forme déjà plus que des sons dégonflés dans ma bouche. Quelque chose a voulu parler à travers moi mais il eût fallu une écoute plus fine, une main plus exercée que la mienne. Je ne suis toujours que le traducteur infidèle. ${ }^{450}$

Il faut souligner ici la "dictée abrupte» et le "langage absolu», qui trouvent un écho dans les textes de la maturité. Le cri est aussi considéré par le narrateur de La Déchirure comme l'expression somme toute commune de l'angoisse de chacun:

Ce n'est pas tant votre déchirure qui importe, vous savez bien que vous n'avez pas tant d'importance, c'est la déchirure du monde qui effraie. Ce sang qui s'écoule dans le lieu obscur. Les cris que personne n'entend. Les cris qui sont proférés dans le sommeil de tous, lorsqu'on n'a plus la force de remettre le masque qui glisse sur le visage et que la chose terrible approche. ${ }^{451}$

Dans Le Régiment noir, qui est le roman du père, une "histoire d'enfants" (selon Bauchau ${ }^{452}$ ) qui jouent à la guerre, aux amitiés viriles et à l'Amérique du mélange des races, les cris sont principalement ceux des batailles, les hurlements de l'attaque et ceux des lyncheurs - mais également ceux des blessés. Une des expressions lancinantes du roman est le redoublement: "En avant! En avant!» ${ }^{\mathbf{5 3} 3}$ qui caractérise autant la manière dont le récit progresse tambour battant, que le tempo avec lequel les personnages traversent l'Amérique, ses combats, ses conquêtes.

Avant de passer aux ouvres romanesques de la maturité, il convient d'évoquer un texte poétique, La Sourde oreille ou le rêve de Freud, qui recèle en son centre un des cris saillants, poussé en rêve, de l'œuvre de Bauchau. Marc Quaghebeur dit de cet ouvrage qu'il "constitue vraiment un livre charnière entre la période suisse et celle du cycle thébain qui va débuter. Il s'agit aussi d'un véritable livre d'aveux " ${ }^{\mathbf{4 5 4}}$.

448 Ibid., pp. 147, 190, 192.

449 Blanche Reverchon Jouve dit à Henry Bauchau, dans "La circonstance éclatante": "Votre levier, c'est l'écriture», Henry Bauchau, L'Écriture à l'écoute, Arles, Actes Sud, 2000, p. 24.

450 Henry Bauchau, La Déchirure, op. cit., p. 241.

451 Ibid., p. 244.

452 C'est ce qu'il écrit dans une préface rédigée à la troisième personne du singulier, en 2000, placée en tête de la réédition du roman et intitulée "D'une relecture»: "C'est beaucoup plus tard, après avoir longuement vécu sur la route avec Edipe et Antigone, qu'il a compris que sous le masque guerrier du Régiment noir, il n'avait raconté qu'une histoire d'enfants. L'histoire aventureuse d'une Amérique d'autrefois en soulevant un peu le voile amnésique qui recouvre les joies, les épreuves, les traversées de la nuit et le courageux soleil des enfances", Henry Bauchau, Le Régiment noir, Arles, Actes Sud, 2004, coll. Babel, p. 13.

453 Ibid., pp. 170, 240, 302.

454 Marc Quaghebeur, "Revisitées, les confessions de La Sourde oreille inventent pour l'écrivain la 
[Freud] dit: vous êtes un malade imaginaire.

Et je pousse un immense cri!

Un cri, qui monte, qui retentit à travers toute ma vie.

Je crie et je m’éveille - mais me suis-je éveillé vraiment? - je me découvre épouvanté.

[...]

Pourquoi la profonde lignée a-t-elle à travers toi poussé ce cri de peur?

Il fallait écouter, entendre encore le Docteur Freud.

Il s'apprêtait, tu l'as bien vu - c'est alors que tu as crié - il s'approchait pour te parler.

De ton enfance ou de ta mère, dont tu es sorti un matin sans regard et déjà tout empli d'images.

Pourquoi n'as-tu pas écouté ce qui venait après? Et craignais-tu vraiment d'être un malade imaginaire? ${ }^{455}$

On peut souligner ici que le cri, faisant partie du rêve, participe de cette écriture de la vision qu'est l'œuvre bauchalienne. L'attention aux rêves, à leur signification, à l'espace du non-dit qu'ils mettent en scène, rencontre ici le cri et ce non-dit qu'il implique.

Si La Sourde oreille est un texte charnière, il l'est aussi par cette écriture du cri. Nous avons vu, dans le premier temps de l'œuvre, qui est celui de la maturation de l'écriture tant poétique que dramatique ou romanesque, que le motif du cri s'étend à l'originel, au domaine amoureux, au désir de conquête et de puissance, et enfin à la découverte de soi, par l'inconscient, le rêve et la psychanalyse. Mais dans les œuvres ultérieures, il gagne en intensité et en potentiel sémique, en puissance d'exutoire et de sidération.

Le motif s'intensifie en effet dans les récits d'CEdipe et d'Antigone, mais également dans une des scènes marquantes du Boulevard périphérique. Les cris d'Antigone sont mémorables, notamment celui qu'elle pousse dans Edipe sur la route pour empêcher le suicide de son père au chapitre VI, intitulé «Le refus d'Antigone»:

Elle le secoue, elle crie: "CEdipe, tu ne peux pas mourir, tu le pouvais autrefois, tu le pouvais à Thèbes. Ici, tu n'en as plus le droit. "Elle se redresse, elle hurle comme une pythie: "Tu n'en as plus le droit. Je te le refuse, à cause... à cause de moi, Antigone! „456

La comparaison avec la pythie mérite une attention particulière. Antigone se trouve ainsi investie d'un rôle de prophétesse, dans une visée épique, avant même d'avancer dans le destin qui lui a été forgé par la mythologie et que Bauchau

légende de son futur", dans Pierre Halen, Raymond Michel et Monique Michel (dir.), Henry Bauchau, une poétique de l'espérance, Berne, Peter Lang, 2004, p. 133.

455 Henry Bauchau, La Sourde oreille ou le rêve de Freud, Lausanne, L'Aire, 1981, p. 21.

456 Henry Bauchau, Edipe sur la route, Arles, Actes Sud, 1990, "Babel», p. 127. 
réécrit. Dans le même mouvement, elle se trouve liée au dieu Apollon, l'Apollon pythien, celui-là même qui a tué le serpent Python afin d'installer son oracle à Delphes. Lier Antigone à Apollon, même en la comparant à la pythie, c'est déjà la placer sur la voie de la mesure, d'une certaine forme de justice et de sérénité.

Dans CEdipe sur la route, Edipe crie également à quatre reprises. Le premier hurlement apparaît au chapitre "La Vague», lié à l'accomplissement artistique l'achèvement périlleux de la sculpture de la vague - et rattaché à des événements antérieurs vécus par le personnage: «Il crie, il hurle de colère. C'est ainsi qu'il a dû crier quand il a tué le roi Laïos et ses gardes. [...] Il se tord au bout de la corde et vomit, en criant entre chaque crise. ${ }^{457}$ Clios et Antigone sont témoins de la scène, mais c'est cette dernière qui perçoit la métamorphose d'CEdipe: "À bout de souffle [Antigone] est obligée de s'arrêter, les cris d'CEdipe la chavirent et pourtant elle perçoit qu'ils ont changé de nature. Ils ressemblent à ceux qu'il poussait dans la cour du palais lorsqu'il s'entraînait avec ses gardes. [...] Antigone est sur le cap, CEdipe ne crie plus. ${ }^{458}$ À la fin de la scène, pour marquer l'exploit physique et psychique du héros, ce sont des vivats de victoire, d'exultation, que les trois personnages sont en mesure d'échanger:

[Clios] est triomphant, jubilant et, lorsque EEdipe hurle de sa voix d'airain, il lui fait écho de toute la force de la sienne. Il se tourne vers [Antigone], il la contraint à regarder, à comprendre, à soutenir, elle aussi, de sa violence l'acte qui a eu lieu là. Elle ne peut résister à son regard et elle répond par ses clameurs à celles qu'Edipe ou Zeus profèrent avec la mer. Sybille ou pythie, elle n'est plus qu'une voix qui arrache de son corps son cri le plus extrême tandis que lui ébranle la falaise, de ses outils divins et de l'inconcevable épaule. ${ }^{459}$

Antigone est à nouveau associée à la figure de la prophétesse. On peut insister à cet égard sur le fait que la pythie - tout comme l'artiste - accepte d'être traversée par quelque chose qui dépasse l'entendement, "extrême» et "inconcevable».

Le second cri poussé par Edipe, dans le chapitre "Le solstice d'été", prend également valeur de révélation et d'acceptation de soi. Après la découverte de son destin d'aède, le héros est pénétré par la face sombre de ce don, qui est un hurlement:

Il dresse vers le ciel un masque inattendu, un long museau argenté et il pousse un hurlement qui fait souffrir et se prolonge à l'infini. Beaucoup sentent se hérisser leur échine, car ils entendent le loup le plus antique et qui hurle à la lune. Un loup venu du fond des âges abominables, celui que suivait Apollon avant de devenir le conducteur du char solaire. Le loup qui précédait les rats de la peste et qui anime toujours, dans le cœur des hommes, les puissances de destruction. Tous ceux qui plongent encore leurs racines dans ce sol ancestral se lèvent, pressés par le désir

457 Ibid., p. 154.

458 Ibid., p. 155.

459 Ibid., p. 157. 
de hurler avec CEdipe et de s'unir en meute autour de lui. ${ }^{460}$

On retrouve la référence à Apollon, mais celui d'avant la lumière, qui est une figure inquiétante. Par le hurlement ancestral, Edipe est relié à l'histoire profonde de sa lignée.

Plus loin dans le roman, c'est de l'expérience du cri originel, celui de la naissance, qu'il est question avec la nouvelle mise au monde d'CEdipe par Calliope, dans le chapitre "Calliope et les pestiférés»: "CEdipe hurle en s'agitant sur sa couche comme quelqu'un qui souffre et qui vit encore. Calliope entend ses cris comme ceux d'un tout petit enfant et, laissant retomber ses pieds blessés, elle s'approche de sa tête, la serre contre elle et doucement le berce. " ${ }^{\mathbf{4 6 1}} \mathrm{Si}$ CEdipe crie comme un enfant, Calliope, elle, gémit comme une parturiente:

Vers le matin, Calliope pousse un grand cri. [...] Elle est sur le lit d'CEdipe, à cheval sur ses épaules, et elle l'a retourné sur le ventre. Elle semble faire d'énormes efforts, accompagnés de cris et de plaintes, pour le faire sortir de son corps. [...] un cri plus perçant de Calliope leur fait comprendre la nécessité impérieuse de sortir CEdipe de sous elle. [...] Les cris et les halètements de Calliope continuent, mais ils n'ont plus cet accent d'impuissance et de désespoir. Elle crie: «Il bouge!» en poussant son corps en avant [...] son corps sombre agité par le formidable travail. ${ }^{462}$

Ces trois premiers cris d'Edipe structurent les étapes de la construction de son personnage: sa vie de voyant aveuglé, son don de créateur, son lien aux forces ancestrales et sa renaissance, qui signifie un nouveau pas vers la voyance, même aveugle. La scène, à chaque reprise, indique que la signification se trouve au-delà des mots, dans ce que le corps saisit sans que l'esprit ne le sache. Cela mène à une certaine forme de sagesse, illustrée dans un nouveau cri: CEdipe, sur le point de se faire lapider devant les portes de Thèbes, toujours en compagnie de Clios et d'Antigone, fait éclater une pierre en plein vol, grâce à son cri, avant qu'elle ne l'atteigne.

Restent trois colosses bien décidés à les lapider. Le premier saisit une grosse pierre, la brandit, la lance vers eux, Clios se jette en avant pour protéger Antigone. À ce moment, CEdipe qui s'est levé pousse un cri, la pierre éclate en l'air avec un bruit affreux. Les débris retombent aux pieds du lanceur. Les trois brutes, prises de peur, s'engouffrent dans la ville. [...] «Comment as-tu opéré ce prodige? - Il n’y a pas de prodige, dit CEdipe, c'est un cri que m'a appris en Égypte un officier du pharaon. " La colère de Clios se ranime : "Et si elle n’avait pas éclaté? - Je dois obéir à la route que je ne connais pas. Aujourd'hui, elle passait par ici. - Elle y passait aussi pour moi", dit Antigone. ${ }^{463}$

On observe ici un nouveau type de savoir: la sagesse nommée "Tao» dans

460 Ibid., pp. 187-188.

$461 \mathrm{Ibid}$., p. 237.

462 Ibid., pp. 238-239.

463 Ibid., pp. 252-253. 
Diotime et les lions ${ }^{\mathbf{4 6 4}}$, marquée du sceau de l'Orient. On peut souligner que le cri pour CEdipe ne fait donc pas que surgir et le submerger, lui révélant au passage ses potentialités créatrices, ses racines, les richesses de son être ou les liens qui l'unissent à ses proches, mais qu'il peut également faire l'objet d'un apprentissage, et devenir une arme.

Quant à Antigone, le roman qui porte son nom a eu pour titre de travail Le Cri d'Antigone ${ }^{\mathbf{4 6 5}}$. L'héroïne pousse deux hurlements décisifs. Le premier, c'est celui de la mendiante, qu'elle a appris sur la route, avec Edipe, comme moyen de les nourrir tous deux. Elle le redécouvre pour obtenir des Thébains des dons qui lui permettront de poursuivre son ouvre de soutien aux plus démunis. Sa sour Ismène est stupéfaite en la voyant apparaître généreusement dotée:

Tu as tiré tout ça de ces sourds, de ces avares, c'est incroyable. Comment as-tu fait, Antigone?

- Je ne sais pas. J'ai crié.

- Tu as crié! Toi, Antigone?

- Oui. J'avais honte mais ils m’ont donné. Ils ont dû.

- Ils ont dû! Tu criais quoi?

- Je ne sais pas, ce n'était plus moi qui étais là... Je criais contre la vie ou pour elle. ${ }^{466}$

Remarquons qu'Antigone n'est "plus [...] là " quand elle crie, c'est-à-dire qu'elle n'est plus elle-même, ou qu'elle est dépassée par une entité plus vaste qu'elle. Le chapitre dont est extrait ce passage, intitulé "Le cri», connaît une première version publiée comme récit indépendant, daté de 1993 et inclus dans le recueil Les Vallées du bonbeur profond, publié en 1995. On y trouve déjà la description de ce qui submerge l'héroïne :

Ce n'est plus l'ancien cri [qu'elle peut] pousser ici, il est trop faible pour la ville inexorable où plus personne n'écoute. [...] Il y a une colère, une soudaine fureur qui s'élève, grandit en traversant son corps et produit un cri. Le cri d'un enfant malingre, enfermé, abandonné, qui entrevoit, à travers des siècles ténébreux, l'espérance, l'existence de la clarté. C'est le cri vers la lumière de ceux qui sont nés d'elle et pour elle, mais qui en ont été indéfiniment exilés. ${ }^{467}$

Le hurlement se développe en prenant la dimension d'un cri originel, se fait le porte-voix de tous ceux qui n'ont plus la force d'élever la leur. Ainsi Antigone n'est plus seulement elle-même, mais le peuple des démunis, leur voix pour dire la misère du monde:

464 Henry Bauchau, Diotime et les lions, Arles, Actes Sud, 1991, "Babel», p. 53.

465 Henry Bauchau le mentionne à plusieurs reprises dans son Journal d'Antigone (1989-1997), Arles, Actes Sud, 1999, pp. 234, 276, 282, 283, 293, 300, 311 - c'est-à-dire entre le 11 mai 1993 et le 4 janvier 1994. Par la suite le roman est désigné par son titre définitif.

466 Henry Bauchau, Antigone, Arles, Actes Sud, 1997, "Babel», p. 198.

467 Henry Bauchau, Les Vallées du bonheur profond. Récits, Arles, Actes Sud, 1999, coll. Babel, p. 57. 
Le cri veut s'élever à nouveau, elle tente de le contenir dans son ventre qui se crispe, de le barricader dans sa gorge qui s'étrangle et pourtant il jaillit. Non, il n'y a pas assez de malheurs, de hontes, de crimes, pas assez d'absurdes désastres, de vies détruites, de bonheurs piétinés. Pas assez de sang, d'enfants tués, de destruction, de folie sur terre. Il faut que la chose grandisse, montre enfin au grand jour sa tête hideuse et molle et dévoile sa puanteur. Il ne suffit pas que la chose soit vue, il faut qu'elle soit parlée, plus haut, beaucoup plus haut. Que son terrible langage soit entendu et qu'il déborde, ici et maintenant, puisque le lieu où il devrait être proféré, puisque ce lieu n'existe pas. ${ }^{468}$

La mendiante Antigone n'articule rien mais dit tout; elle dénonce par la violence du surgissement lui-même. C'est là un des traits marquants de l'utilisation du cri par Bauchau: l'insistance du signifiant zéro qui porte un signifié infini. Quant au «lieu qui n'existe pas» mentionné à la dernière ligne, on entrevoit qu'il s'agit de celui qui, à la fin du roman, se présente comme l'espace auquel la mort d'Antigone conduit, dans le relais de sa parole par le chant d'Io - à savoir le théâtre. Dans cette perspective, Bauchau confère au théâtre un rôle d'exutoire (catharsis), mais également de connaissance et d'espérance. C'est la scène de théâtre qui permet que "la chose soit vue», "qu'elle soit parlée», dans la continuation du cri de la protagoniste.

Le second cri marquant du roman Antigone s'élève dans le chapitre intitulé "Le tribunal ", qui voit la condamnation à mort par Créon de la fille d'CEdipe qui a osé rendre de sommaires honneurs funèbres à son frère Polynice. Elle crie simplement "non" pour détourner l'attention de Créon et empêche ainsi Ismène de prendre la parole et de se compromettre, elle et l'enfant qu'elle porte. Ce non à tout ce que représentent son jugement et les lois de Thèbes prend des dimensions fabuleuses:

C'est le non de toutes les femmes que je prononce, que je hurle, que je vomis avec celui d'Ismène et le mien. Ce non vient de bien plus loin que moi, c'est la plainte, ou l'appel qui vient des ténèbres et des plus audacieuses lumières de l'histoire des femmes. [...] Je crie non, rien que non, rien d'autre n'est utile. Non, seul suffit. [...] La violence du non arrache de leurs gonds les portes de la salle, chasse les juges, épouvante les conseillers et les force à fuir, abandonnant sur le sol les médiocres débris de leurs insignes et de leur dignité. Il ne reste plus, face à face, que Créon et moi, je pourrais encore amplifier le cri, ébranler les murs du tribunal et faire s'écrouler sur lui ce monument d'iniquité. ${ }^{469}$

Mais Antigone, qui est encore plus qu'elle-même, magnifiée par une force qui rappelle celle qu'CEdipe manifeste à plusieurs reprises dans Edipe sur la route (avançant littéralement à pas de géant pour atteindre les Hautes Collines, par exemple), finit par maîtriser son cri et refuse l'écroulement de ce tribunal. Elle ne passe pas du côté de la destruction. Elle fait vibrer cette colère, ce

468 Ibid., p. 59.

469 Henry Bauchau, Antigone, op. cit., pp. 318-319. 
refus, mais se résigne ensuite au jugement prononcé. Bauchau écrit : «la colère veut dire espérance ${ }^{\mathbf{4 7 0}}$. Il faut garder cela à l'esprit, ainsi que la déclinaison du cri d'Antigone en trois temps : la prophétesse, la mendiante et l'insoumise, qui tous trois la définissent. Son "non" formidable apparaît comme une grâce, une inspiration fulgurante qui fait d'elle la porte-parole des maux de l'humanité, une figure christique peut-être, mais avant cela, une figure qui fonderait l'essence du théâtre tragique.

Dans Le Boulevard périphérique, alors que le contexte romanesque n'est plus mythique mais historique et contemporain, c'est un même cri de refus, de protection, qui retentit au chapitre IV, à travers le récit que fait Mary au narrateur d'un épisode de l'Occupation:

Arrivées près de la gare, toutes les femmes se sont mises à crier. Je ne sais pas qui a commencé. Ce n'était pas des paroles, pas des injures ou des revendications, rien qu'un cri. Après un moment je me suis aperçue que je criais comme les autres. Tout bas d'abord, puis un peu plus fort, puis très fort. Et les prisonniers se sont mis à crier aussi. On se répondait. [...] Nous avions de plus en plus peur et nous criions de plus en plus fort. [...] Et quand les soldats et les SS sont arrivés avec leurs fusils et leurs matraques, ils se sont trouvés devant des femmes qui n'attaquaient pas, qui ne faisaient pas mine de se défendre. Qui criaient, qui criaient rien du tout. De plus en plus fort, dans quelle panique! Et les voix s'élevaient vers l'aigu [...] Nous hurlions rien du tout, les femmes sont comme ça, on se foutait des «Vive» et des "À bas». On criait à cause de quelque chose qui n'aurait jamais dû se vivre, quelque chose de honteux. Oui, quelque chose de honteux pour eux, qui étaient aussi des fils de femmes, des maris, des amants, des pères, qu'est-ce que je sais, moi. [...] Et on criait. [...] Les femmes on sait que ça crie, mais ça c'était un cri comme ils n'en avaient jamais entendu et qui leur remuait les tripes et tout. ${ }^{471}$

À ce qui n'articule aucune parole sont opposées des paroles de peur qui perdent en force:

On s'est toutes retournées vers les ouvriers en criant: "Arrêtez!» [...] nous avions perdu notre belle unité: on ne poussait plus un seul cri formidable face aux ennemis. [...] Et on poussait des cris, les petits cris de bonne femme qu'ils attendaient: "Arrêtez. Vous êtes fous. Ils vont tirer." ${ }^{472}$

Mais après ce moment de faiblesse, les femmes se ressaisissent et reprennent à l'unisson le cri sans paroles, celui de la vie:

Nous avons crié de nouveau toutes ensemble. Crié rien du tout. Jean-Jacques et les autres allaient partir pour l'Allemagne. Déportés comme tant d'autres. Mais avec une chance. Une chance de s'enfuir, une chance de revenir. Vivants en tout

470 Henry Bauchau, La Déchirure, op. cit., p. 132; voir également dans L'Écriture à l'écoute, op. cit., p. 52 .

471 Henry Bauchau, Le Boulevard périphérique, Arles, Actes Sud, 2008, pp. 38-40.

472 Ibid., pp. 44-45. 
cas, aujourd'hui vivants. Et les autres, nous et les hommes, vivants aussi. Ça valait la peine de crier, non?473

La force de ce chapitre est de proposer un récit d'espérance, qui peut paraître dérisoire au vu du contexte, mais grâce auquel l'humanité se trouve affermie. En contraste avec le cri d'Antigone, qui était celui d'une personne portant celui de tous, cette scène de l'Occupation présente le cri de toutes, qui deviennent une, et qui ne dit "rien du tout", mais qui puise sa force dans ce signifiant zéro. Ces hurlements, tant ceux d'CEdipe et d'Antigone que celui de ces femmes, constituent des actes qui prennent force de sidération - ils médusent ou bouleversent ceux qui les entendent - et changent le cours de l'histoire. Le cri permet l'œuvre, relie aux ancêtres, obtient l'aumône, refuse la propagation de la mort, ou soutient la révolte. Ces scènes métamorphosent leurs protagonistes, transfigurés par l'expérience et la force dont ils sont soudain investis.

Régis Lefort, dans son ouvrage L'Originel dans l'œuvre d'Henry Bauchau, estime que le chant signifie le "dépassement de la voix individuelle " ${ }^{44}$, dès lors que grâce à lui "de nombreux personnages de Bauchau entrent en communication avec un au-delà ${ }^{475}$. Nous avons vu que le chant, tant pour CEdipe que pour Antigone, se présente comme une annonce ou un prolongement du cri et qu'il entre en résonance avec un "en-deçà» des personnages, avec ces "propres profondeurs, qui pour une part sont traversées par le discours collectif "476, selon l'expression de Pierre Halen. Si les premiers textes de Bauchau, écrits durant la période suisse de sa maturation littéraire, offrent des indices de la présence du cri, les textes de la maturité font du cri autant une expérience du dépassement de soi, dans le temps et dans l'espace, qu'une expérience des limites du langage ${ }^{477}$.

"Comme toujours chez Bauchau, on n'en reste pas à l'indicible, on va tenter de dire, et prendre le risque de ne pas simplifier "478, souligne Pierre Halen. C'est là qu'une écriture tournée vers la vision, la maturation lente par le rêve, le martèlement de la vie et des lectures, prend tout son sens. Cette écriture court le risque, dans une simplicité apparente, d'exprimer ce dont on ne peut pas parler. Elle ne

\footnotetext{
473 Ibid., p. 47.

474 Régis Lefort, L'Originel dans l'œuvre d'Henry Bauchau, Paris, Champion, 2007, p. 263.

475 Ibid., p. 268.

476 Pierre Halen, "Une poétique de l'espérance?», dans Henry Bauchau, une poétique de l'espérance, op. cit., p. 4.

477 Les limites imparties à cette étude ne nous permettent pas de développer l'analyse pour l'ensemble de l'œuvre romanesque. Notons par exemple que dans Déluge, le cri se fait également l'expression du dépassement de soi, et touche à la folie du personnage de Florian. Celle-ci se manifeste dans la peinture, mais après le cri et comme pour s'en approprier les échos: "Je suis traversé par un grand cri brûlant, mon corps sort de moi et se tord sur le sol, coupé en deux, coupé en mille peut-être. [...] "Dans quel état vous vous êtes mis? Si on ne vous avait pas fait tout de suite une transfusion, vous y restiez. Vous avez beaucoup crié.” (Henry Bauchau, Déluge, Arles, Actes Sud, 2010, pp. 114-115.) 478 Pierre Halen, "À propos du "monde ancien" dans l'œuvre d'Henry Bauchau: une approche du Boulevard périphérique», dans Revue internationale Henry Bauchau: L'écriture à l'écoute, $\mathrm{n}^{\circ} 1,2008$, p. 72 .
} 
retire donc pas la confiance placée dans la langue. Elle s'obstine, malgré tout, à être à l'écoute de la nécessité intérieure. Comme le dit Bauchau dans son Journal d'Antigone: "Antigone devait donc lancer son cri qui n'accepte pas et transmet l'intraitable espérance. "479

Odile Cornuz

Université de Neuchâtel

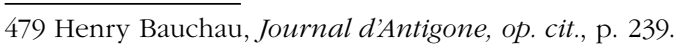

